\title{
Observation of a Liquid Phase with an Orthorhombic Orientational Order
}

\author{
D. Kaminski, P. Poodt, E. Aret, N. Radenovic, and E. Vlieg \\ IMM Department of Solid State Chemistry, Radboud University Nijmegen, Toernooiveld 1, 6525 ED Nijmegen, The Netherlands
}

(Received 25 October 2005; published 7 February 2006)

\begin{abstract}
Using surface $\mathrm{x}$-ray diffraction, we have determined the structure of liquid Bi monolayers on $\mathrm{Cu}(111)$ for a range of coverages. By combining diffuse scattering data from the liquid with information from the substrate scattering, the ordering properties of Bi have been fully determined. Even though the substrate is hexagonal, we find that the liquid does not show hexatic order but has an orthorhombic orientational order that occurs in three domains. Simultaneously, Bi has partial solidlike properties, even well above its melting point.
\end{abstract}

DOI: 10.1103/PhysRevLett.96.056102

PACS numbers: 68.08.De, 61.10.Nz, 68.15.+e

Since the description of 2D melting and the prediction of a hexatic phase with long-range orientational and shortrange positional order by Kosterlitz, Thouless, Halperin, Nelson, and Young [1], such phenomena have been observed in several experimental systems. Most results are from liquid crystalline materials, for which freestanding films are nearly ideal realizations of a 2D system [2], and new details about the phase behavior continue to be reported [3]. Hexatic(like) phases have further been observed in the presence of a substrate, e.g., rare gases on graphite [4], $\mathrm{Pb}$ on $\mathrm{Ge}(111)$ [5], or $\mathrm{Pb}$ on $\mathrm{Cu}(111)$ [6]. The substrate in these cases has hexagonal symmetry, but its influence is not entirely clear [7]. This liquid ordering is not only of fundamental interest but is also relevant for solid-liquid interfaces as found during lubrication, wetting, and crystal growth [8].

Here we present the high-temperature behavior of a (quasi)liquid $\mathrm{Bi}$ monolayer on $\mathrm{Cu}(111)$. Given the similarity with $\mathrm{Pb}-\mathrm{Cu}(111)$, a hexaticlike phase is expected, but instead we find that the orientational order shows orthorhombic symmetry. As far as we are aware, such a type of liquid ordering has not been observed before.

Following the pioneering work of Delamare and Rhead on the $\mathrm{Bi}-\mathrm{Cu}(111)$ system [9], we recently reported the structure of various room-temperature phases of this system as a function of Bi coverage [10]. At low coverage, a surface alloy is formed, but at higher coverages the two metals do not mix. This and the fact that Bi layers can be prepared with high purity in ultrahigh vacuum make Bi$\mathrm{Cu}(111)$ a good system to study the properties of the liquid monolayer.

We have employed surface x-ray diffraction to study the structure and the melting behavior of the Bi layer [11]. Below the melting transition, the Bi layer is in registry with the $\mathrm{Cu}(111)$ substrate and will contribute to the substrate diffraction rods. This interference makes it possible to determine the structure of the surface in detail. After melting, the Bi-Bi pair correlation in the liquid monolayer will give rise to a cylinder of diffuse scattering ("liquid ring") whose azimuthal intensity distribution depends on the orientational order in the liquid [5]. It will be isotropic for a true liquid but has a sixfold symmetry for a hexatic phase. However, even above the melting temperature, a fraction of the liquid may still be in registry with the substrate and will then continue to contribute to the substrate diffraction rods $[12,13]$. By combining the possibility to measure both the diffuse liquid scattering and the contribution to the substrate, $\mathrm{x}$-ray diffraction can thus quantitatively determine the ordering properties of the liquid and the role that the substrate plays in this.

The measurements were performed at the DUBBLE beam line at the European Synchrotron Radiation Facility in Grenoble, France [14], using an x-ray energy of $16 \mathrm{keV}$. The results were fully reproducible over two separate experimental runs. The experimental setup allows us to accurately control both the temperature and the $\mathrm{Bi}$ coverage. The error in absolute temperature is about $20^{\circ} \mathrm{C}$, but the relative error is around $2{ }^{\circ} \mathrm{C}$. The cylindrical $\mathrm{Cu}(111)$ crystal had a diameter of $8 \mathrm{~mm}$ and was polished within $\sim 0.3^{\circ}$ of the crystallographic (111) plane [15]. For experimental details, we refer to our previous paper [10].

For convenience, we use a (111) surface unit cell expressed in the bulk lattice vectors of $\mathrm{Cu}$ as follows:

$\mathbf{a}_{1}=\frac{1}{2}[10 \overline{1}]_{\text {cubic }}, \quad \mathbf{a}_{2}=\frac{1}{2}[\overline{1} 10]_{\text {cubic }}, \quad \mathbf{a}_{3}=[111]_{\text {cubic }}$

with

$$
\left|\mathbf{a}_{1}\right|=\left|\mathbf{a}_{2}\right|=\frac{1}{2} \sqrt{2} a, \quad\left|\mathbf{a}_{3}\right|=\sqrt{3} a,
$$

and $a=3.61 \AA$ the lattice constant of $\mathrm{Cu}$. The corresponding reciprocal lattice vectors $\mathbf{b}_{j}$ are defined by $\mathbf{a}_{i} \cdot \mathbf{b}_{j}=$ $2 \pi \delta_{i j}$. The momentum transfer is $\boldsymbol{Q}=h \mathbf{b}_{1}+k \mathbf{b}_{2}+l \mathbf{b}_{3}$, with $(h k l)$ the diffraction indices. With this choice for the unit cell, the index $l$ is the direction perpendicular to the surface. Crystal truncation rods have integer $(h k)$ indices; the extra rods arising from a surface reconstruction have fractional $(h k)$ indices.

We first had to determine the phase diagram over the relevant coverage and temperature range, because the first reported phase diagram was not accurate enough [9]. The results are shown in Fig. 1(a). At room temperature, the system shows a $(\sqrt{3} \times \sqrt{3}) R 30^{\circ}$ reconstruction at an ideal 

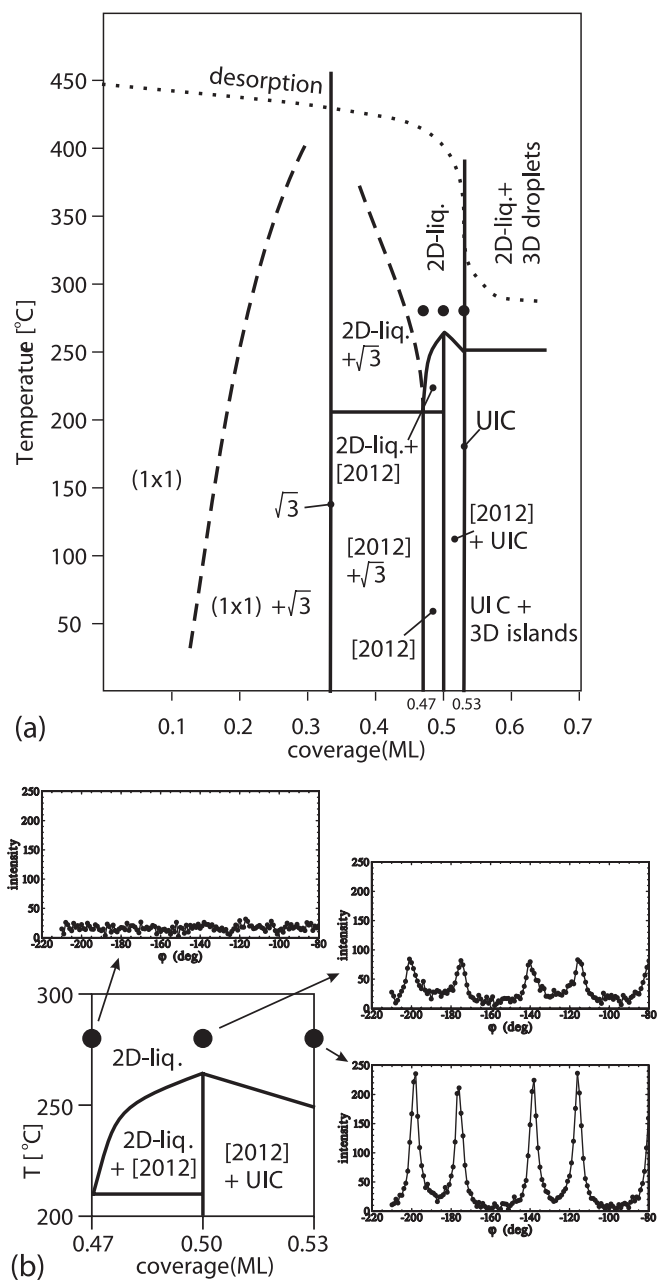

FIG. 1. (a) Phase diagram of a Bi monolayer on $\mathrm{Cu}(111)$. The nomenclature is explained in the text. The dotted line indicates the temperature above which desorption is too rapid to perform our type of experiments. (b) A detailed view of the phase diagram around $0.5 \mathrm{ML} \mathrm{Bi}$ and $250{ }^{\circ} \mathrm{C}$, together with backgroundsubtracted azimuthal scans through the diffuse scattering rings at Bi coverages of $0.47,0.50$, and $0.53 \mathrm{ML}$ and at a temperature of $280^{\circ} \mathrm{C}$. The solid circles in the phase diagrams indicate the conditions for the azimuthal scans.

coverage of $1 / 3$ monolayer (ML), a

$$
\left(\begin{array}{ll}
2 & 0 \\
1 & 2
\end{array}\right)
$$

reconstruction at $0.5 \mathrm{ML}$, and an uniaxial-incommensurate phase at $0.527 \mathrm{ML}[10]$. From now on, we will denote these phases by the shorthand notation $\sqrt{3}$, [2012], and UIC, respectively. These phases are highly ordered, with a correlation length near $900 \AA$ as determined from the corresponding fractional-order reflections. At intermediate coverages, we usually find coexistence of two phases, but the [2012] phase is found to be stable over a small coverage range from 0.47 to $0.5 \mathrm{ML}$. By observing at which temperature the fractional-order peaks disappear, the melting (or disordering) point of the different surface phases is determined. The melting point of the [2012] phase depends strongly on coverage and increases from $210^{\circ} \mathrm{C}$ at $0.47 \mathrm{ML}$ to $265^{\circ} \mathrm{C}$ at $0.5 \mathrm{ML}$. Such coverage dependence has been observed in other (sub)monolayer films as well [16]. The UIC phase melts at $250^{\circ} \mathrm{C}$. At high temperatures, the desorption rate of $\mathrm{Bi}$ becomes appreciable, and this prevented us from determining the melting point for the $\sqrt{3}$ phase.

In the following, we will focus on the coverage regime between 0.47 and $0.53 \mathrm{ML}$. The nature of the disordered phase above the melting point can be determined from the diffuse liquid scattering by combining radial and azimuthal scans [see Fig. 2(a)]. We investigated this systematically for a temperature of $280^{\circ} \mathrm{C}$, well above the phase transition temperature over the entire coverage range discussed here. At a coverage of $0.47 \mathrm{ML}$, we found an isotropic liquid ring at a $Q_{\|}$value of $1.93 \AA^{-1}$. The cylindrical shape of this scattering (and, thus, the 2D nature of the liquid) was confirmed by performing radial scans for different values of the perpendicular momentum transfer. The ori-

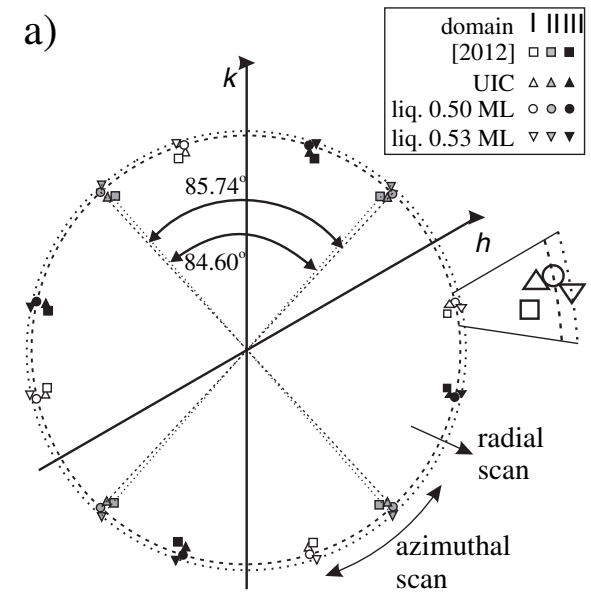

b)

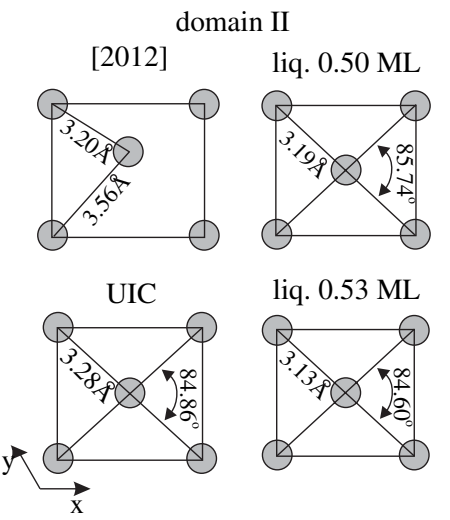

FIG. 2. (a) Positions in reciprocal space for selected reflections of the [2012] and the UIC phases at room temperature and for the maxima in the liquid ring at $280^{\circ} \mathrm{C}$ for coverages of 0.50 and $0.53 \mathrm{ML}$. In all cases, the pattern arises from three rotational domains on the $\mathrm{Cu}(111)$ surface. (b) The local structure in real space of the four structures shown in (a) for domains with orientation II. 
entational order in the liquid is determined from an azimuthal scan while observing the maximum intensity in the radial direction. The background-subtracted result is shown in Fig. 1(b), where a scan range a little over $120^{\circ}$ is shown. The threefold symmetry of the substrate means that the pattern is repeated over intervals of $120^{\circ}$ (as confirmed by wider scans). At $0.47 \mathrm{ML}$ coverage the liquid is clearly isotropic. For increasing coverage, however, the liquid becomes anisotropic as shown by the azimuthal scans for a coverage of 0.50 and $0.53 \mathrm{ML}$ and measured at the maximum intensity observed for $Q_{\|}$values of 1.97 and $2.01 \AA^{-1}$, respectively. The location of the ring, thus, shifts to higher $Q_{\|}$values as a function of coverage, which agrees with the smaller nearest-neighbor distance expected at higher densities. At a coverage of $0.53 \mathrm{ML}$, the liquid is found to be highly anisotropic, with some directions having almost zero intensity. The azimuthal position of the maxima is also found to shift slightly. The most surprising observation, however, is the number of maxima. We find four maxima over a $120^{\circ}$ azimuth, corresponding to 12 maxima in a full $360^{\circ}$ scan. This is twice the number expected for a hexatic phase. The strong peaks, on the other hand, clearly show that there is long-range orientational order, just as in the hexatic phase. The orientational order at $0.53 \mathrm{ML}$ is larger than for $0.50 \mathrm{ML}$, even though this phase is farther above the melting point. Other points on the phase diagram show comparable behavior, e.g., $0.47 \mathrm{ML} \mathrm{Bi}$ at $220^{\circ} \mathrm{C}$ shows similar modulation as $0.50 \mathrm{ML}$ at $280^{\circ} \mathrm{C}$.

From the peak width of the radial scans, we can estimate the radial correlation length $L_{\mathrm{rad}}=2 / \Delta Q_{\mathrm{rad}, \mathrm{fwhm}}$. For the nonisotropic cases, this depends on the orientation, and the largest $L_{\text {rad }}$ corresponds with the intensity maxima. Similarly, the azimuthal correlation length $L_{\mathrm{az}}$ can be derived from the azimuthal scans. These results are listed in Table I and show that both correlation lengths increase with coverage and that the maximum value is near $15 \AA$, about 5 times a typical $\mathrm{Bi}-\mathrm{Bi}$ distance.

Next we will show that a plausible explanation for the observed peaks is that they arise from three liquid domains with orientational order along four directions each. Figure 2(a) shows the location of all 12 peaks in reciprocal space as derived from the azimuthal scans and the $p 3 m 1$ symmetry of the substrate for the coverages of 0.50 and $0.53 \mathrm{ML}$. At room temperature, the surface shows a [2012] and a UIC phase, respectively, at these coverages. In reciprocal space, several lattice points of these phases are found to be very close to the liquid peaks, e.g., $(0.50,0.25)$ for the [2012] phase and $(0.527,0.235)$ for the UIC phase. For both room-temperature phases, the full pattern near the ring consists of three domains with four lattice points each, and these are indicated by different gray scales in Fig. 2(a). This strongly suggests that (i) the peaks from the liquid phase arise from a local structure that is very similar to that in the room-temperature phase and (ii) that this local structure is present in three domains as dictated by the
TABLE I. Parameters for $\mathrm{Bi}$ derived for three different $\mathrm{Bi}$ coverages on $\mathrm{Cu}(111)$ at $280^{\circ} \mathrm{C}$. The height is with respect to a top fcc $\mathrm{Cu}$ atom. The substrate shows no significant relaxation. $B_{\perp}=2 \AA^{2}$ for all $\mathrm{Bi}$ atoms.

$\begin{array}{lll}0.47 \mathrm{ML} & 0.50 \mathrm{ML} & 0.53 \mathrm{ML}\end{array}$

From liquid ring

$L_{\text {rad }}(\AA)$

7

$L_{\mathrm{az}}(\AA)$

0

$8-10$

9-17

From interference with substrate rods

Liquid fraction

\begin{tabular}{lccc}
$\Delta z(\AA)$ & $0.44 \pm 0.03$ & $0.44 \pm 0.03$ & $0.44 \pm 0.03$ \\
$B_{\|}\left(\AA^{2}\right)$ & 1000 & 1000 & 1000 \\
Occupancy & $0.01 \pm 0.07$ & $0.21 \pm 0.08$ & $0.29 \pm 0.08$ \\
fcc $=$ hcp fraction & & & \\
$\Delta z(\AA)$ & $0.35 \pm 0.03$ & $0.35 \pm 0.03$ & $0.35 \pm 0.03$ \\
$B_{\|}\left(\AA^{2}\right)$ & $14 \pm 5$ & $9 \pm 5$ & $2 \pm 5$ \\
Occupancy & $0.22 \pm 0.01$ & $0.13 \pm 0.05$ & $0.11 \pm 0.05$ \\
Total coverage $(\mathrm{ML})$ & $0.45 \pm 0.07$ & $0.47 \pm 0.09$ & $0.51 \pm 0.09$ \\
$\chi^{2}$ & 2.48 & 2.67 & 2.79 \\
\hline \hline
\end{tabular}

underlying substrate. From the four peaks in the azimuthal scans in Fig. 1, only two are thus from the same domain. Based on this, each Bi atom is fourfold coordinated in the liquid. The liquid, thus, has orthorhombic orientational order.

The dimensions of this local structure can be derived from the peak positions in reciprocal space. Figure 2(b) compares this local structure (for a single domain) with the structure of the ordered phases at room temperature [10]. In calculating the nearest-neighbor distance $a_{0}$ in the liquid, an uncertainty arises. Using a 2D Fourier transform, the relation between peak position $Q$ in reciprocal space and $a_{0}$ for an isotropic 2D liquid is given by [5] $a_{0}=$ $1.12 \times 2 \pi / Q$. The present liquid, however, is far from isotropic, and the more simple relation $a_{0}=2 \pi / Q$ appears to be more appropriate. The values used in Fig. 2(b) are obtained using the latter equation. As the figure shows, the local liquid structure we derive in this manner closely resembles the structure of the solid phases [2012] and UIC.

The Bi layer shows more order than the azimuthal anisotropy in the pair-correlation function, because Fig. 3 shows that the liquid contributes to the (10) substrate rods (with in-plane momentum transfer) as well. Compared with the curve calculated for a bulk-terminated crystal (short-dashed line), the data for $0.50 \mathrm{ML} \mathrm{Bi}$ at $280{ }^{\circ} \mathrm{C}$ (solid circles) still show a significant contribution from the bismuth. (The data for the other liquid coverages are not shown but are similar.) For comparison, the data for the [2012] reconstructed phase is also shown.

The fact that we observe both a liquid ring and $\mathrm{Bi}$ modulated $\mathrm{Cu}(111)$ rods shows that $\mathrm{Bi}$ has both liquid and solid properties [13]. We can model this situation by including both solid and liquid $\mathrm{Bi}$ atoms. The liquid frac- 


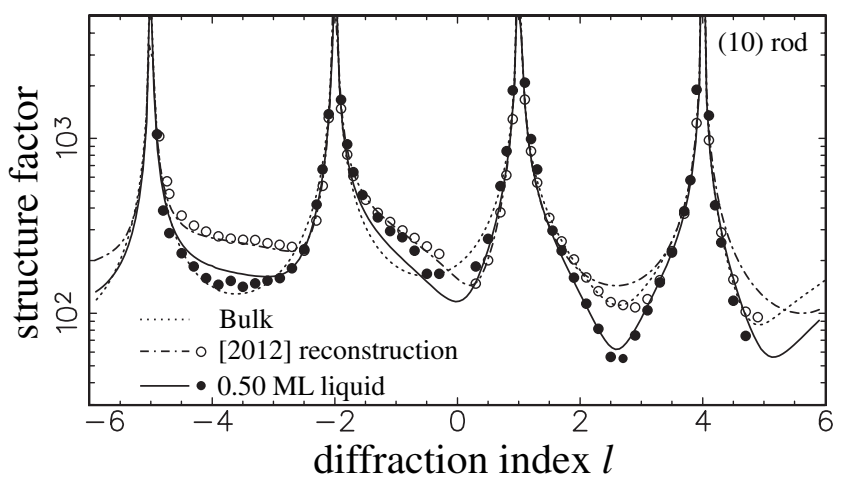

FIG. 3. Structure factor amplitudes along the (10) rod for the [2012] reconstruction, the liquid phase at $0.50 \mathrm{ML}$, and, for comparison, a bulk-terminated $\mathrm{Cu}(111)$ substrate. Symbols indicate data; the curves represent the best-fit models.

tion has no in-plane order, corresponding to very large inplane Debye-Waller parameters $B_{\|}$(we use a value of $1000 \AA^{2}$ ), while the solid fraction has small $B_{\|}$values. By fitting the data for the (00) and (10) rods simultaneously, the different fractions can be determined. There are three possible high-symmetry positions for the solid fraction: an on-top site and two hollow sites, one at an fcc and one at an hcp location. We find that the on-top site is not occupied and disregard this in the following. The model yields excellent fits to the data for all coverages; the result for the (10) rod and 0.50 ML is shown as the solid curve in Fig. 3, and the fit parameters are listed in Table I. From these fits, we find that the solid fraction occupies fcc and hcp sites equally and that this fraction is decreasing for increasing coverage. At the same time, the liquid fraction is increasing, as expected from the increasing intensity in the liquid ring as a function of coverage. From the intensity in the liquid ring, the ratio of the liquid fraction is estimated to be 1:2:3 for $0.47: 0.50: 0.53 \mathrm{ML}$, respectively. This is just outside the error bar of the fitted value for 0.47 ML, likely because of the correlations between the various fit parameters. Nevertheless, the total $\mathrm{Bi}$ coverage from the fits follows the actual values quite closely.

From the combined data of diffuse scattering and the substrate rods, the following picture emerges of the liquid structure at $280^{\circ} \mathrm{C}$. For all three coverages, a significant fraction of the molten $\mathrm{Bi}$ atoms is located near the two different hollow sites of the substrate. At the lowest coverage $(0.47 \mathrm{ML})$, there is little steric hindrance and the azimuthal distribution is isotropic. For higher coverages, the $\mathrm{Bi}$ atoms start to interact more strongly and locally adapt a fourfold coordinated structure that closely resembles the ordered phases at low temperature. The interaction with the substrate gives rise to three orientational domains, explaining the 12 observed peaks in the diffuse scattering. The increased steric hindrance causes a smaller fraction of the $\mathrm{Bi}$ atoms to be near the hollow substrate sites and leads to a larger fraction with liquid behavior. The high mobility in the layer means that there is no strong distinction between "solid" and "liquid" Bi atoms: The measured solid fraction indicates only the time fraction that an atom can be associated with a hollow substrate site.

The balance between Bi-Bi interactions and the influence of the substrate thus lead to a peculiar behavior of three liquid domains with orthorhombic orientational order that is very different from the hexatic order found in similar systems. We are aware of only one report of nonhexatic liquid order, but that was for computer simulations of confined multilayers [17]. In several earlier experiments of liquids on substrates, only the diffuse scattering has been observed [4-6]. It would, therefore, be interesting to measure the substrate rods for these cases as well, since this more directly reveals the role of the substrate and possible solidlike properties in the liquid.

We thank the DUBBLE staff for their assistance, W. Szweryn for technical support, H. Knops for stimulating discussions, and W. J.P. van Enckevort for a careful reading of the manuscript. This work is supported by the Council for Chemical Sciences of the Netherlands Organization for Scientific Research (CW-NWO).

[1] J. M. Kosterlitz and D. J. Thouless, J. Phys. C 6, 1181 (1973); B. I. Halperin and D. R. Nelson, Phys. Rev. Lett. 41, 121 (1978); A. P. Young, Phys. Rev. B 19, 1855 (1979).

[2] C. F. Chou et al., Science 280, 1424 (1998); W. H. de Jeu, B. I. Ostrovskii, and A. N. Shalaginov, Rev. Mod. Phys. 75, 181 (2003).

[3] C. Y. Chao et al., Phys. Rev. Lett. 93, 247801 (2004).

[4] R. J. Birgeneau and P. M. Horn, Science 232, 329 (1986).

[5] F. Grey et al., Phys. Rev. B 41, 9519 (1990).

[6] B. H. Muller, T. Schmidth, and M. Henzler, Surf. Sci. 376, 123 (1997).

[7] N. Greiser et al., Phys. Rev. Lett. 59, 1706 (1987).

[8] B. Bhushan, J. N. Israelachvili, and U. Landman, Nature (London) 374, 607 (1995); E. Johnson, Science 296, 477 (2002); E. Vlieg, Surf. Sci. 500, 458 (2002).

[9] F. Delamare and G. E. Rhead, Surf. Sci. 35, 185 (1973).

[10] D. Kaminksi et al., Surf. Sci. 575, 233 (2005).

[11] R. Feidenhans'l, Surf. Sci. Rep. 10, 105 (1989); I. K. Robinson and D. J. Tweet, Rep. Prog. Phys. 55, 599 (1992).

[12] S. A. de Vries, P. Goedtkindt, P. Steadman, and E. Vlieg, Phys. Rev. B 59, 13301 (1999).

[13] M.F. Reedijk et al., Phys. Rev. B 64, 033403 (2001).

[14] M. Borsboom et al., J. Synchrotron Radiat. 5, 518 (1998).

[15] The copper crystal was provided by the Surface Preparation Laboratory, Zaandam, The Netherlands.

[16] R. D. Etters, B. Kuchta, and J. Belak, Phys. Rev. Lett. 70, 826 (1993); M. F. Reedijk et al., Phys. Rev. Lett. 90, 056104 (2003).

[17] A. Vishnyakov and A. V. Neimark, J. Chem. Phys. 118, 7585 (2003). 